\title{
New Oleanan-Type Triterpene and Cincholic Acid Glycosides from Peruvian "Uña de Gato" (Uncaria tomentosa)
}

\author{
Mariko Kitajima, ${ }^{a}$ Ken-ichiro Hashimoto, ${ }^{a}$ Manuel Sandoval, ${ }^{b}$ Norio Aimi, ${ }^{a}$ and \\ Hiromitsu TAKAYAMA ${ }^{*, a}$ \\ ${ }^{a}$ Graduate School of Pharmaceutical Sciences, Chiba University; 1-33 Yayoi-cho, Inage-ku, Chiba 263-8522, Japan: and \\ ${ }^{b}$ Center for Research on Amazonian Natural Products, Universidad Nacional Agraria de la Selva; Apartado 156, Tingo \\ Maria, Peru. $\quad$ Received June 1, 2004; accepted August 2, 2004
}

\begin{abstract}
A new oleanan-type triterpene (1) and three new cincholic acid glycosides (2-4) were isolated from Peruvian "Uña de Gato" (Cat's claw, plant of origin: Uncaria tomentosa), a traditional herbal medicine in Peru. Their structures were determined by spectroscopic analysis.
\end{abstract}

Key words triterpene; Uncaria tomentosa; Uña de Gato (Cat's claw); Rubiaceae

The Peruvian traditional herbal medicine, "Uña de Gato" (Cat's claw), ${ }^{1,2)}$ is used for treating various ailments, including arthritis, inflammation and cancer. The plants of origin of "Uña de Gato" are Uncaria tomentosa (WILlD.) D.C. and U. guianensis (AuBL.) GMEL. (Rubiaceae). To date, chemical $^{3-5}$ ) and biological studies ${ }^{6,7)}$ have been conducted by many researchers. Our recent pharmacological studies on oxindole alkaloids, which are the constituents of this herbal medicine, suggested that pteropodine and isopteropodine, which are Heteroyohimbine-type oxindole alkaloids, act as positive modulators of muscarinic $\mathrm{M}_{1}$ and $5-\mathrm{HT}_{2}$ receptors, ${ }^{8)}$ and rhynchophylline and isorhynchophylline, which are Corynanthé-type oxindole alkaloids, act as noncompetitive antagonists of the $N$-methyl-D-aspartate (NMDA) receptor in Xenopus oocytes. ${ }^{9)}$ We have reported the isolation of new triterpenoids ${ }^{10,11)}$ and alkaloids ${ }^{12,13)}$ from Peruvian "Uña de Gato" (plant of origin: Uncaria tomentosa). As a continuation of our chemical study, we isolated one new oleanan-type triterpene (1) and three new cincholic acid glycosides (2-4) from Peruvian "Uña de Gato", which are described herein.

From the $\mathrm{MeOH}$ extract of "Uña de Gato" (plant of origin: Uncaria tomentosa), four new triterpenes (1-4) were isolated together with five known quinovic acid glycosides. The structures of the known compounds were deduced from spectroscopic data and confirmed by comparison with reported data.

The high resolution (HR)-FAB-MS spectrum of 1 measured in the positive ion mode gave a quasi-molecular ion peak at $\mathrm{m} / \mathrm{z} 503.3343\left([\mathrm{M}+\mathrm{H}]^{+}\right)$that corresponded to the molecular formula $\mathrm{C}_{30} \mathrm{H}_{47} \mathrm{O}_{6}\left(m / z\right.$ 503.3372). The ${ }^{1} \mathrm{H}-\mathrm{NMR}$ spectrum $\left(\mathrm{CD}_{3} \mathrm{OD}\right)$ showed five singlets due to six methyl groups at $\delta 1.44,1.06,0.96(6 \mathrm{H}), 0.93$ and 0.78 , one broad singlet at $\delta 3.07$, signals assignable to two methine protons bearing a hydroxyl group at $\delta 3.26(\mathrm{brd}, J=3.9 \mathrm{~Hz}$ ) and 3.54 (dd, $J=11.2,4.4 \mathrm{~Hz}$ ), one pair of doublets due to a hydroxymethyl group at $\delta 3.51$ and 3.38 (each d, $J=10.7 \mathrm{~Hz}$ ), and one signal of an olefinic proton at $\delta 5.36(\mathrm{brt})$. In the ${ }^{13} \mathrm{C}$ NMR spectrum $\left(\mathrm{CD}_{3} \mathrm{OD}\right)$, thirty carbons, including one carboxyl carbon at $\delta 182.3$, two $s p^{2}$ carbons of a trisubstituted olefin at $\delta 144.2$ and 124.5 , two methine carbons bearing a hydroxyl group at $\delta 82.4$ and 72.4 , one hydroxymethyl carbon at $\delta 66.1$, and six methyl carbons $(\delta 28.7,25.7,25.0$, $18.1,17.3,12.8)$, were observed. Therefore, 1 was deduced to be an oleanan-type triterpenoid possessing two secondary hydroxyl groups, one hydroxymethyl group and one carboxyl group. The HMBC cross-peaks between the oxymethine proton at $\delta 3.54$ and the methyl carbon at $\delta 12.8$ and between both the methine proton at $\delta 2.61$ and the methyl protons at $\delta 1.06$ and the oxymethine carbon at $\delta 72.4$ indicated the existence of a hydroxyl group at C-3, as common triterpenes. Furthermore, a signal assignable to a ketone carbon was observed at $\delta 215.8$ in the ${ }^{13} \mathrm{C}$-NMR spectrum. HMBC correlations were observed between the ketone carbon $(\delta$ 215.8) and a singlet proton assignable to $\mathrm{H}-5$ ( $\delta$ 2.61), and between the same carbon and methylene protons assignable to $\mathrm{H}_{2}-7$ ( $\delta 2.69$ and 1.77 , each $\mathrm{d}$ ), indicating that the ketone was located at C-6. Cross-peaks between the hydroxymethyl protons and C-3 $(\delta$ 72.4), C-4 ( $\delta$ 42.4), C-5 ( $\delta$ 59.1) and methyl carbon $(\delta$ 12.8) indicated that the hydroxymethyl group was located at C-4. In the differential NOE experiment, irradiation of the methyl protons located at $\mathrm{C}-4$ ( $\delta$ 1.06) led to enhancement of the signal intensities of the $\mathrm{H}-25$ methyl protons at $\delta 0.96(5 \%)$ and the hydroxymethyl protons at $\delta 3.51(3 \%)$ and $\delta 3.38(2 \%)$, indicating that the

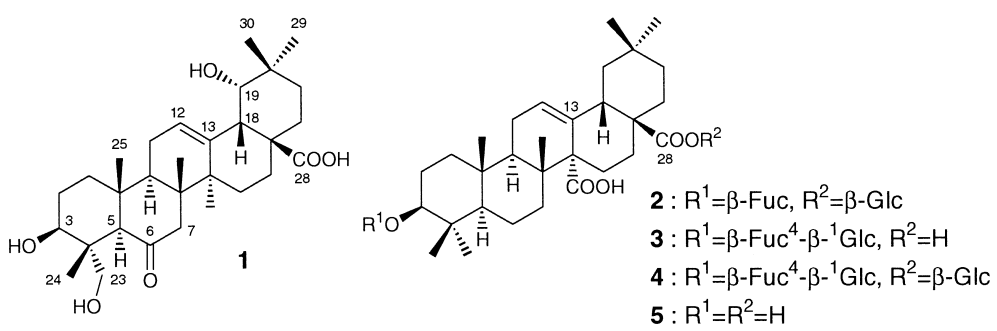

Fig. 1 
hydroxymethyl group at C-4 was $\alpha$-oriented; C-23 was oxidized to hydroxymethyl. HMBC cross-peaks between $\mathrm{H}-18$ $(\delta$ 3.07) and both the carboxyl carbon $(\delta$ 182.3) and the olefinic carbons $(\delta$ 144.2) demonstrated that $\mathrm{C}-28$ was oxidized to a carboxylic acid. Irradiation of the olefinic proton $(\delta 5.36)$ led to $4 \%$ enhancement of the signal intensity of $\mathrm{H}-19(\delta 3.26)$ in the differential NOE experiment, indicating that the hydroxyl group at $\mathrm{C}-19$ was $\alpha$-oriented. From the above data, the structure of 1 was deduced to be $3 \beta, 19 \alpha, 23-$ trihydroxy-6-oxo-olean-12-en-28-oic acid.

The HR-FAB-MS spectrum of $\mathbf{2}$ measured in the negative ion mode gave a quasi-molecular ion peak at $\mathrm{m} / \mathrm{z} 793.4348$ $\left([\mathrm{M}-\mathrm{H}]^{-}\right)$that corresponded to the molecular formula $\mathrm{C}_{42} \mathrm{H}_{65} \mathrm{O}_{14}(\mathrm{~m} / \mathrm{z} 793.4375)$. Acid hydrolysis of 2 gave cincholic acid (5), ${ }^{14)}$ D-glucose and D-fucose. The ${ }^{1} \mathrm{H}-\mathrm{NMR}$ spectrum of 2 showed six singlets assignable to the methyl groups of the triterpene aglycon at $\delta 1.00\left(\mathrm{H}_{3}-23\right), 0.95\left(\mathrm{H}_{3}-\right.$ 25), $0.91\left(\mathrm{H}_{3}-30\right), 0.87\left(\mathrm{H}_{3}-26\right), 0.86\left(\mathrm{H}_{3}-29\right)$ and $0.82\left(\mathrm{H}_{3}-\right.$ $24)$, one olefinic proton signal at $\delta 5.65(\mathrm{H}-12)$ and one characteristic proton signal due to $\mathrm{H}-18$ in oleanan-type triterpenes at $\delta$ 2.90. In addition, signals assignable to two $\beta$-linked anomeric protons at $\delta 5.40\left(\mathrm{~d}, J=7.9 \mathrm{~Hz}, \mathrm{H}-1^{\prime \prime \prime}\right)$ and $4.22\left(\mathrm{~d}, J=7.3 \mathrm{~Hz}, \mathrm{H}-1^{\prime}\right)$ and one doublet methyl signal of fucose at $\delta 1.24\left(\mathrm{H}_{3}-6^{\prime}\right)$ were observed. The ${ }^{13} \mathrm{C}-\mathrm{NMR}$ spectrum $\left(\mathrm{CD}_{3} \mathrm{OD}\right)$ showed forty-two carbons, including one carboxyl carbon at $\delta 179.9(\mathrm{C}-27)$, one ester carbonyl carbon at $\delta 178.0(\mathrm{C}-28)$, two $s p^{2}$ carbons of a trisubstituted olefin at $\delta 137.4(\mathrm{C}-13)$ and $127.8(\mathrm{C}-14)$, two anomeric carbons at $\delta 107.1\left(\mathrm{C}-1^{\prime}\right)$ and $95.6\left(\mathrm{C}-1^{\prime \prime \prime}\right)$, and six methyl carbons at $\delta 33.5$ (C-29), 28.5 (C-23), 24.0 (C-30), 19.0 (C-26) and 16.9 (C-24, C-25). The sugar linkages were deduced from NMR chemical shifts and HMBC experiments. From the chemical shifts of the anomeric protons and carbons, one sugar was found to be attached through an ether linkage and the other sugar unit was attached through an ester linkage.

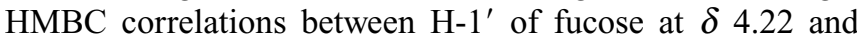
C-3 of the aglycon at $\delta 90.6$ and between H-1"' of glucose at $\delta 5.40$ and the C-28 ester carbonyl carbon at $\delta 178.0$ revealed that fucose and glucose were attached to C-3 and $\mathrm{C}-28$, respectively. Therefore, the structure of $\mathbf{2}$ was deduced to be cincholic acid $3 \beta-O-\beta$-D-fucopyranosyl-28- $O-\beta$-D-glucopyranosyl ester.

The HR-FAB-MS spectrum of $\mathbf{3}$ measured in the negative ion mode gave a quasi-molecular ion peak at $\mathrm{m} / \mathrm{z} 793.4348$ $\left([\mathrm{M}-\mathrm{H}]^{-}\right)$that corresponded to the molecular formula $\mathrm{C}_{42} \mathrm{H}_{65} \mathrm{O}_{14}(\mathrm{~m} / z$ 793.4375), which was the same as that of 2 . Acid hydrolysis of 3 gave cincholic acid (5), D-glucose and D-fucose. The ${ }^{1} \mathrm{H}-\mathrm{NMR}$ spectrum showed two doublets with a large coupling constant due to anomeric protons at $\delta 4.54$ $\left(J=7.6 \mathrm{~Hz}, \mathrm{H}-1^{\prime \prime}\right)$ and $4.23\left(J=7.6 \mathrm{~Hz}, \mathrm{H}-1^{\prime}\right)$, one doublet methyl signal of fucose at $\delta 1.29\left(\mathrm{H}_{3}-6^{\prime}\right)$, six methyl singlets $(\delta 1.01,0.96,0.92,0.88,0.86,0.82)$ and one olefinic proton signal $\left(\delta\right.$ 5.63). The ${ }^{13} \mathrm{C}-\mathrm{NMR}$ spectrum $\left(\mathrm{CD}_{3} \mathrm{OD}\right)$ showed two carboxyl carbons at $\delta 182.1$ and 180.0 , two $s p^{2}$ carbons of a trisubstituted olefin at $\delta 138.1$ and 127.3, two anomeric carbons at $\delta 107.0\left(\mathrm{C}-1^{\prime}\right)$ and $105.9\left(\mathrm{C}-1^{\prime \prime}\right)$ and six methyl carbons at $\delta 33.6,28.5,24.0,18.9,17.1$ and 16.8. From the above data, 3 was deduced to be cincholic acid possessing two $\beta$-linked sugar units attached through ether linkages. The sugar linkages were deduced by HMBC experiments. The
Table 1. ${ }^{13} \mathrm{C}-\mathrm{NMR}$ Data for $\mathbf{1}-\mathbf{4}$ in $\mathrm{CD}_{3} \mathrm{OD}$

\begin{tabular}{|c|c|c|c|c|}
\hline & 1 & 2 & 3 & 4 \\
\hline 1 & 39.7 & 39.9 & 39.9 & 39.9 \\
\hline 2 & 27.0 & 27.1 & 27.0 & 27.0 \\
\hline 3 & 72.4 & 90.6 & 90.8 & 90.8 \\
\hline 4 & 42.4 & 40.1 & 40.1 & 40.1 \\
\hline 5 & 59.1 & 57.1 & 57.0 & 57.0 \\
\hline 6 & 215.8 & 19.3 & 19.3 & 19.3 \\
\hline 7 & 51.7 & 37.8 & 37.8 & 37.8 \\
\hline 8 & 47.7 & 40.7 & 40.6 & 40.7 \\
\hline 9 & a) & 48.3 & 48.3 & 48.3 \\
\hline 10 & 44.1 & 37.9 & 37.9 & 37.9 \\
\hline 11 & 25.1 & 24.0 & 23.9 & 24.0 \\
\hline 12 & 124.5 & 127.8 & 127.3 & 127.8 \\
\hline 13 & 144.2 & 137.4 & 138.1 & 137.4 \\
\hline 14 & 43.0 & 57.2 & 57.3 & 57.3 \\
\hline 15 & $29.5^{b)}$ & 25.6 & 25.6 & 25.6 \\
\hline 16 & 28.4 & 25.1 & 25.2 & 25.1 \\
\hline 17 & 46.7 & a) & a) & a) \\
\hline 18 & 45.2 & 44.6 & 44.8 & 44.6 \\
\hline 19 & 82.4 & 44.6 & 44.7 & 44.6 \\
\hline 20 & 36.1 & 31.5 & 31.6 & 31.5 \\
\hline 21 & $29.4^{b)}$ & 34.7 & 34.8 & 34.7 \\
\hline 22 & 33.9 & 32.7 & 33.4 & 32.7 \\
\hline 23 & 66.1 & 28.5 & 28.5 & 28.5 \\
\hline 24 & 12.8 & 16.9 & 17.1 & 17.1 \\
\hline 25 & 17.3 & 16.9 & 16.8 & 16.9 \\
\hline 26 & 18.1 & 19.0 & 18.9 & 19.0 \\
\hline 27 & 25.7 & 179.9 & 180.0 & 180.0 \\
\hline 28 & 182.3 & 178.0 & 182.1 & 178.0 \\
\hline 29 & 28.7 & 33.5 & 33.6 & 33.5 \\
\hline 30 & 25.0 & 24.0 & 24.0 & 24.0 \\
\hline Fuc-1' & & 107.1 & 107.0 & 107.0 \\
\hline $2^{\prime}$ & & 72.9 & 73.5 & 73.5 \\
\hline $3^{\prime}$ & & 75.3 & $75.8^{b)}$ & $75.8^{b)}$ \\
\hline $4^{\prime}$ & & 73.1 & 82.4 & 82.3 \\
\hline $5^{\prime}$ & & 71.6 & 71.2 & 71.2 \\
\hline $6^{\prime}$ & & 17.0 & 17.4 & 17.4 \\
\hline Glc-1" & & & 105.9 & 105.9 \\
\hline $2^{\prime \prime}$ & & & $75.9^{b)}$ & $75.9^{b)}$ \\
\hline $3^{\prime \prime}$ & & & 78.1 & 78.1 \\
\hline $4^{\prime \prime}$ & & & 71.6 & 71.6 \\
\hline $5^{\prime \prime}$ & & & 78.3 & 78.2 \\
\hline $6^{\prime \prime}$ & & & 62.9 & 62.9 \\
\hline Glc-1"' & & 95.6 & & 95.6 \\
\hline $2^{\prime \prime \prime}$ & & 73.9 & & 73.9 \\
\hline $3^{\prime \prime \prime}$ & & 78.3 & & 78.3 \\
\hline $4^{\prime \prime \prime}$ & & 71.2 & & 71.2 \\
\hline $5^{\prime \prime \prime}$ & & 78.7 & & 78.7 \\
\hline $6^{\prime \prime \prime}$ & & 62.5 & & 62.5 \\
\hline
\end{tabular}

a) Under $\mathrm{CD}_{3} \mathrm{OD}$ signal. b) Interchangeable.

HMBC correlation between $\mathrm{H}-1^{\prime}$ of fucose at $\delta 4.23$ and $\mathrm{C}-3$ of the aglycon at $\delta 90.8$ revealed that fucose was attached to C-3 of the aglycon. The HMBC correlation between both $\mathrm{H}-1^{\prime \prime}$ of glucose at $\delta 4.54$ and methyl protons $\left(\mathrm{H}_{3}-6^{\prime}\right)$ of fucose at $\delta 1.29$ and $\mathrm{C}-4^{\prime}$ of fucose at $\delta 82.4$ revealed that a terminal glucose was attached to $\mathrm{C}-4^{\prime}$ of an inner fucose. Therefore, the structure of $\mathbf{3}$ was deduced to be cincholic acid $3 \beta$ - $O$ - $\beta$-D-glucopyranosyl- $(1 \rightarrow 4)-\beta$-D-fucopyranoside.

The HR-FAB-MS spectrum of $\mathbf{4}$ measured in the positive ion mode gave a sodiated molecular ion peak at $\mathrm{m} / \mathrm{z}$ $979.4908\left([\mathrm{M}+\mathrm{Na}]^{+}\right)$that corresponded to the molecular formula $\mathrm{C}_{48} \mathrm{H}_{76} \mathrm{O}_{19} \mathrm{Na}(\mathrm{m} / \mathrm{z} 979.4878)$. Acid hydrolysis of 4 gave cincholic acid (5), D-glucose, and D-fucose. The existence of three sugar units was revealed by the three $\beta$-linked anomeric proton signals at $\delta 5.40$ (d, $\left.J=8.2 \mathrm{~Hz}, \mathrm{H}-1^{\prime \prime \prime}\right), 4.54$ 
(d, $\left.J=7.6 \mathrm{~Hz}, \mathrm{H}-1^{\prime \prime}\right)$ and $4.23\left(\mathrm{~d}, J=7.3 \mathrm{~Hz}, \mathrm{H}-1^{\prime}\right)$ in the ${ }^{1} \mathrm{H}-$ NMR spectrum. The ${ }^{13} \mathrm{C}$-NMR spectrum was very similar to that of $\mathbf{3}$ except for the signals due to one extra glucose unit including the anomeric carbon at $\delta 95.6\left(\mathrm{C}-1^{\prime \prime \prime}\right)$. HMBC correlations revealed that $\mathbf{4}$ has the same sugar chain as $\mathbf{3}$ at C-3 position and an extra glucose unit at C-28 through an ester linkage. Therefore, the structure of $\mathbf{4}$ was deduced to be cincholic acid $3 \beta$ - $O$ - $\beta$-D-glucopyranosyl-( $(1 \rightarrow 4)-\beta$-D-fucopyranosyl-28- $O$ - $\beta$-D-glucopyranosyl ester.

\section{Experimental}

General Experimental Procedures Optical rotation: JASCO DIP-140. IR: JASCO FT/IR-230. ${ }^{1} \mathrm{H}$ - and ${ }^{13} \mathrm{C}-\mathrm{NMR}$ spectra: at $500\left({ }^{1} \mathrm{H}-\mathrm{NMR}\right)$ and 125.65 $\left({ }^{13} \mathrm{C}-\mathrm{NMR}\right) \mathrm{MHz}$, respectively, JEOL JNM A-500. FAB-MS and HRFAB-MS: JEOL JMS-HX110. TLC: Precoated silica gel $60 \mathrm{~F}_{254}$ plates (Merck, $0.25 \mathrm{~mm}$ thick). Column chromatography: Silica gel 60 (Merck, 70 -230 mesh for open column chromatography and 230 - 400 mesh for flash column chromatography), DIAION HP20 (Mitsubishi Chemical), Sephadex LH-20 (Pharmacia Biotech). MPLC: C. I. G. prepacked column CPS-HS-221-05 $\left(\mathrm{SiO}_{2}\right)$ and CPO-HS-221-20 (ODS) (Kusano Kagakukikai). HPLC: Shodex RSpak DC-613 (Showa Denko).

Plant Material "Uña de Gato" used in this study was imported from Peru through Coperunix Japan, Inc. (Tokyo, Japan) in 1996. The plant of origin was confirmed to be Uncaria tomentosa (stem and stem bark) by the company. A voucher specimen (no. 961001) was deposited at the herbarium of the Graduate School of Pharmaceutical Sciences, Chiba University.

Extraction and Isolation "Uña de Gato" ( $835 \mathrm{~g}$, dry weight) was extracted with hot $\mathrm{MeOH}$ six times $(11 \times 6)$ to give the $\mathrm{MeOH}$ extract (total: $95 \mathrm{~g}$ ). Fifty grams of the $\mathrm{MeOH}$ extract (1st, 2nd and 3rd extracts were combined) was subjected to column chromatography on DIAION HP20 to give seven fractions, as described previously. ${ }^{10)}$ Fraction D (12.44 g) eluted with $\mathrm{MeOH}$ was separated by $\mathrm{SiO}_{2}$ gel open column chromatography ( $\mathrm{MeOH} / \mathrm{CHCl}_{3}$ gradient). The $30-35 \% \mathrm{MeOH} / \mathrm{CHCl}_{3}$ eluate was purified by flash column chromatography and MPLC $\left(\mathrm{SiO}_{2}, 10 \% \mathrm{MeOH} / \mathrm{CHCl}_{3}\right)$, followed by $\mathrm{SiO}_{2}$ flash column chromatography ( $n$-hexane/AcOEt gradient) to give new compound $1(3.5 \mathrm{mg})$. Fraction E $(13.43 \mathrm{~g})$ eluted with $\mathrm{MeOH} / \mathrm{H}_{2} \mathrm{O}=7: 3$ on a DIAION HP20 column gave quinovic acid $3 \beta-O-\beta$ D-glucopyranosyl-( $1 \rightarrow 4)$ - $\beta$-D-fucopyranosyl-( $28 \rightarrow 1)$ - $O$ - $\beta$-D-glucopyranosyl ester $^{15)}(1078.7 \mathrm{mg})$.

The $n$-BuOH extract $(25 \mathrm{~g})$, which was obtained from a portion $(40 \mathrm{~g})$ of the $\mathrm{MeOH}$ extract (4th, 5th and 6th extracts were combined), was subjected to column chromatography on DIAION HP20 to give six fractions, as described previously. $\left.{ }^{12}\right)$ Fraction $\mathrm{J}(9.50 \mathrm{~g})$ eluted with $\mathrm{MeOH}$ was purified by $\mathrm{SiO}_{2}$ gel flash column chromatography $\left(\mathrm{MeOH} / \mathrm{CHCl}_{3}\right.$ gradient) and MPLC $\left(\mathrm{SiO}_{2}, 20 \% \mathrm{MeOH} / \mathrm{CHCl}_{3}\right)$, followed by MPLC (ODS, $\mathrm{H}_{2} \mathrm{O}-\mathrm{MeOH}$, $2: 5)$ to afford new compound $\mathbf{3}(28.8 \mathrm{mg})$. Quinovic acid $3 \beta$ - $O$ - $\beta$-D-glucopyranosyl- $(1 \rightarrow 4)-\beta$-D-fucopyranoside $\left.{ }^{15}\right)(186.9 \mathrm{mg})$, quinovic acid $3 \beta-O-$ $\beta$-D-glucopyranoside ${ }^{16)}(9.1 \mathrm{mg})$ and quinovic acid $3 \beta$ - $O$ - $\beta$-D-glucopyranosyl-(1 $\rightarrow 4)-\beta$-L-rhamnopyranoside ${ }^{15)}(19.8 \mathrm{mg})$ were also obtained from fraction J. Fraction I $\left(5.24 \mathrm{~g}\right.$ ) eluted with $\mathrm{MeOH} / \mathrm{H}_{2} \mathrm{O}=7: 3$ on a DIAION HP20 column gave new cincholic acid glycosides $2(45.3 \mathrm{mg})$ and 4 $(85.4 \mathrm{mg})$, together with quinovic acid $3 \beta$ - $O$ - $\beta$-D-glucopyranosyl- $(1 \rightarrow 4)-\beta$ -

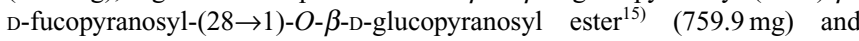
quinovic acid $3 \beta$ - $O$ - $\beta$-D-quinovopyranosyl- $(28 \rightarrow 1)-O-\beta$-D-glucopyranosyl ester ${ }^{17)}(14.8 \mathrm{mg})$. Fraction I was separated by $\mathrm{SiO}_{2}$ gel flash column chromatography ( $\mathrm{MeOH} / \mathrm{CHCl}_{3}$ gradient). The $40 \% \mathrm{MeOH} / \mathrm{CHCl}_{3}$ eluate $(1.56 \mathrm{~g})$ was purified by Sephadex LH-20 chromatography $(\mathrm{MeOH})$ and by MPLC (ODS, $\mathrm{H}_{2} \mathrm{O}-\mathrm{MeOH}, 1: 1.5$ ) repeatedly to afford new glycoside 2. The combined fraction of $50-90 \% \mathrm{MeOH} / \mathrm{CHCl}_{3}$ and $\mathrm{MeOH}$ eluates was separated by Sephadex LH-20 chromatography $(\mathrm{MeOH})$ and by MPLC (ODS, $\mathrm{H}_{2} \mathrm{O}-\mathrm{MeOH}, 1: 1.5$ ) repeatedly to give new glycoside 4.

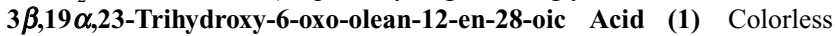
amorphous powder. $[\alpha]_{\mathrm{D}}^{25}+17.2^{\circ}(c=0.11, \mathrm{MeOH})$; selected ${ }^{1} \mathrm{H}-\mathrm{NMR}$ $\left(\mathrm{CD}_{3} \mathrm{OD}, 500 \mathrm{MHz}\right) \delta: 5.36(1 \mathrm{H}$, brt $, J=3.5 \mathrm{~Hz}, \mathrm{H}-12), 3.54(1 \mathrm{H}, \mathrm{dd}$, $J=11.2,4.4 \mathrm{~Hz}, \mathrm{H}-3$ ), 3.51 and 3.38 (each $1 \mathrm{H}, \mathrm{d}, J=10.7 \mathrm{~Hz}, \mathrm{H}_{2}-23$ ), 3.26 $(1 \mathrm{H}$, br d, $J=3.9 \mathrm{~Hz}, \mathrm{H}-19), 3.07(1 \mathrm{H}$, br s, H-18), $2.69(1 \mathrm{H}, \mathrm{d}, J=12.3 \mathrm{~Hz}$, H-7), $2.61(1 \mathrm{H}, \mathrm{s}, \mathrm{H}-5), 2.38(1 \mathrm{H}, \mathrm{dd}, J=10.4,7.0 \mathrm{~Hz}, \mathrm{H}-9), 2.31(1 \mathrm{H}, \mathrm{m})$, 2.11 and 2.01 (each $\left.1 \mathrm{H}, \mathrm{m}, \mathrm{H}_{2}-11\right), 1.77(1 \mathrm{H}, \mathrm{d}, J=12.3 \mathrm{~Hz}, \mathrm{H}-7), 1.44(3 \mathrm{H}$, $\left.\mathrm{s}, \mathrm{H}_{3}-27\right), 1.06\left(3 \mathrm{H}, \mathrm{s}, \mathrm{H}_{3}-24\right), 0.96\left(6 \mathrm{H}, \mathrm{s}, \mathrm{H}_{3}-25, \mathrm{H}_{3}-30\right), 0.93\left(3 \mathrm{H}, \mathrm{s}, \mathrm{H}_{3}-\right.$ 29), 0.78 (3H, s, $\left.\mathrm{H}_{3}-26\right) ;{ }^{13} \mathrm{C}$-NMR: Table 1 ; FAB-MS (NBA, positive) $\mathrm{m} / \mathrm{z}$ $503[\mathrm{M}+\mathrm{H}]^{+}$; HR-FAB-MS (NBA, positive) $m / z 503.3343[\mathrm{M}+\mathrm{H}]^{+}$(Calcd for $\mathrm{C}_{30} \mathrm{H}_{47} \mathrm{O}_{6}$, 503.3372).

Cincholic Acid $3 \boldsymbol{\beta}$ - $O$ - $\boldsymbol{\beta}$-D-Fucopyranosyl-28- $\boldsymbol{O}-\boldsymbol{\beta}$-D-glucopyranosyl Ester (2) Colorless amorphous powder. $[\alpha]_{\mathrm{D}}^{25}+36.0^{\circ}(c=1.21, \mathrm{MeOH})$ IR (KBr) $v_{\max } 3367,2942,1733,1699,1067 \mathrm{~cm}^{-1}$; selected ${ }^{1} \mathrm{H}-\mathrm{NMR}$ $\left(\mathrm{CD}_{3} \mathrm{OD}, 500 \mathrm{MHz}\right) \delta: 5.65(1 \mathrm{H}, \mathrm{m}, \mathrm{H}-12), 5.40\left(1 \mathrm{H}, \mathrm{d}, J=7.9 \mathrm{~Hz}, \mathrm{H}-1^{\prime \prime \prime}\right)$, $4.22\left(1 \mathrm{H}, \mathrm{d}, J=7.3 \mathrm{~Hz}, \mathrm{H}-1^{\prime}\right), 3.08(1 \mathrm{H}, \mathrm{dd}, J=11.4,4.4 \mathrm{~Hz}, \mathrm{H}-3), 2.90(1 \mathrm{H}$, br dd, $J=13.6,3.5 \mathrm{~Hz}, \mathrm{H}-18), 1.24\left(3 \mathrm{H}, \mathrm{d}, J=6.4 \mathrm{~Hz}, \mathrm{H}_{3}-6^{\prime}\right), 1.00\left(3 \mathrm{H}, \mathrm{s}, \mathrm{H}_{3}-\right.$ 23), $0.95\left(3 \mathrm{H}, \mathrm{s}, \mathrm{H}_{3}-25\right), 0.91\left(3 \mathrm{H}, \mathrm{s}, \mathrm{H}_{3}-30\right), 0.87\left(3 \mathrm{H}, \mathrm{s}, \mathrm{H}_{3}-26\right), 0.86(3 \mathrm{H}$, $\left.\mathrm{s}, \mathrm{H}_{3}-29\right), 0.82\left(3 \mathrm{H}, \mathrm{s}, \mathrm{H}_{3}-24\right), 0.71(1 \mathrm{H}, \mathrm{d}, J=11.3 \mathrm{~Hz}, \mathrm{H}-5) ;{ }^{13} \mathrm{C}-\mathrm{NMR}$ : Table 1; FAB-MS (NBA+MeOH, negative) $\mathrm{m} / z 793[\mathrm{M}-\mathrm{H}]^{-}$; HR-FAB-MS (NBA+MeOH, negative) $m / z 793.4348[\mathrm{M}-\mathrm{H}]^{-}$(Calcd for $\mathrm{C}_{42} \mathrm{H}_{65} \mathrm{O}_{14}$, 793.4375).

Cincholic Acid $3 \boldsymbol{\beta}$ - $\boldsymbol{O}$ - $\boldsymbol{\beta}$-D-Glucopyranosyl-(1 $\rightarrow$ 4)- $\boldsymbol{\beta}$-D-fucopyranoside (3) Colorless amorphous powder. $[\alpha]_{\mathrm{D}}^{25}+31.2^{\circ}(c=0.82, \mathrm{MeOH})$; IR $(\mathrm{KBr}) v_{\max } 3320,2942,1687,1069 \mathrm{~cm}^{-1}$; selected ${ }^{1} \mathrm{H}-\mathrm{NMR}\left(\mathrm{CD}_{3} \mathrm{OD}\right.$, $500 \mathrm{MHz}) \delta: 5.63(1 \mathrm{H}, \mathrm{m}, \mathrm{H}-12), 4.54\left(1 \mathrm{H}, \mathrm{d}, J=7.6 \mathrm{~Hz}, \mathrm{H}-1^{\prime \prime}\right), 4.23(1 \mathrm{H}, \mathrm{d}$, $\left.J=7.6 \mathrm{~Hz}, \mathrm{H}-1^{\prime}\right), 3.08(1 \mathrm{H}, \mathrm{dd}, J=11.4,4.5 \mathrm{~Hz}, \mathrm{H}-3), 2.89(1 \mathrm{H}, \mathrm{brdd}$, $J=13.4,3.4 \mathrm{~Hz}, \mathrm{H}-18), 1.29\left(3 \mathrm{H}, \mathrm{d}, J=6.6 \mathrm{~Hz}, \mathrm{H}_{3}-6^{\prime}\right), 1.01\left(3 \mathrm{H}, \mathrm{s}, \mathrm{H}_{3}-23\right)$, $0.96\left(3 \mathrm{H}, \mathrm{s}, \mathrm{H}_{3}-25\right), 0.92\left(3 \mathrm{H}, \mathrm{s}, \mathrm{H}_{3}-30\right), 0.88\left(3 \mathrm{H}, \mathrm{s}, \mathrm{H}_{3}-26\right), 0.86(3 \mathrm{H}, \mathrm{s}$, $\left.\mathrm{H}_{3}-29\right), 0.82\left(3 \mathrm{H}, \mathrm{s}, \mathrm{H}_{3}-24\right), 0.72(1 \mathrm{H}, \mathrm{d}, J=11.5 \mathrm{~Hz}, \mathrm{H}-5) ;{ }^{13} \mathrm{C}-\mathrm{NMR}$ : Table 1; FAB-MS (NBA, negative) $m / z 793[\mathrm{M}-\mathrm{H}]^{-}$; HR-FAB-MS (NBA, negative) $m / z 793.4348[\mathrm{M}-\mathrm{H}]^{-}\left(\right.$Calcd for $\mathrm{C}_{42} \mathrm{H}_{65} \mathrm{O}_{14}, 793.4375$ ).

Cincholic Acid $3 \boldsymbol{\beta}$ - $\boldsymbol{O}$ - $\boldsymbol{\beta}$-D-Glucopyranosyl- $(1 \rightarrow 4)-\boldsymbol{\beta}$-D-fucopyranosyl28- $\boldsymbol{O}$ - $\boldsymbol{\beta}$-D-glucopyranosyl Ester (4) Colorless amorphous powder. $[\alpha]_{\mathrm{D}}^{25}$ $+26.0^{\circ}(c=1.01, \mathrm{MeOH}) ; \mathrm{IR}(\mathrm{KBr}) v_{\max } 3340,2941,1731,1698$, $1069 \mathrm{~cm}^{-1}$; selected ${ }^{1} \mathrm{H}-\mathrm{NMR}\left(\mathrm{CD}_{3} \mathrm{OD}, 500 \mathrm{MHz}\right) \delta: 5.64(1 \mathrm{H}, \mathrm{m}, \mathrm{H}-12)$, $5.40\left(1 \mathrm{H}, \mathrm{d}, J=8.2 \mathrm{~Hz}, \mathrm{H}-1^{\prime \prime \prime}\right), 4.54\left(1 \mathrm{H}, \mathrm{d}, J=7.6 \mathrm{~Hz}, \mathrm{H}-1^{\prime \prime}\right), 4.23(1 \mathrm{H}, \mathrm{d}$, $\left.J=7.3 \mathrm{~Hz}, \mathrm{H}-1^{\prime}\right), 3.08(1 \mathrm{H}, \mathrm{dd}, J=11.4,4.4 \mathrm{~Hz}, \mathrm{H}-3), 2.90(1 \mathrm{H}, \mathrm{brdd}$, $J=13.6,3.5 \mathrm{~Hz}, \mathrm{H}-18), 1.29\left(3 \mathrm{H}, \mathrm{d}, J=6.4 \mathrm{~Hz}, \mathrm{H}_{3}-6^{\prime}\right), 1.00\left(3 \mathrm{H}, \mathrm{s}, \mathrm{H}_{3}-23\right)$, $0.95\left(3 \mathrm{H}, \mathrm{s}, \mathrm{H}_{3}-25\right), 0.91\left(3 \mathrm{H}, \mathrm{s}, \mathrm{H}_{3}-30\right), 0.87\left(3 \mathrm{H}, \mathrm{s}, \mathrm{H}_{3}-26\right), 0.86(3 \mathrm{H}, \mathrm{s}$, $\left.\mathrm{H}_{3}-29\right), 0.81\left(3 \mathrm{H}, \mathrm{s}, \mathrm{H}_{3}-24\right), 0.71(1 \mathrm{H}, \mathrm{d}, J=11.3 \mathrm{~Hz}, \mathrm{H}-5) ;{ }^{13} \mathrm{C}-\mathrm{NMR}$ : Table 1 ; FAB-MS (glycerol, positive) $\mathrm{m} / \mathrm{z} 979 \quad[\mathrm{M}+\mathrm{Na}]^{+}$; HR-FAB-MS (NBA+NaCl, positive) $m / z 979.4908[\mathrm{M}+\mathrm{Na}]^{+}\left(\right.$Calcd for $\mathrm{C}_{48} \mathrm{H}_{76} \mathrm{O}_{19} \mathrm{Na}$, 979.4878).

Acid Hydrolysis of 2 A solution of $2(4.7 \mathrm{mg})$ in $5 \%$ aqueous $\mathrm{H}_{2} \mathrm{SO}_{4}$ $(0.25 \mathrm{ml})$ and 1,4-dioxane $(0.25 \mathrm{ml})$ was heated at $115^{\circ} \mathrm{C}$ for $2 \mathrm{~h}$ under $\mathrm{Ar}$. Water was added to the reaction mixture and the entire mixture was extracted with AcOEt. The organic layer was washed with water, dried over $\mathrm{MgSO}_{4}$ and evaporated. The residue was purified by silica gel open column chromatography ( $\mathrm{MeOH} / \mathrm{CHCl}_{3}$ gradient) to afford cincholic acid $(5,1.6 \mathrm{mg})$, as identified from ${ }^{1} \mathrm{H}$ - and ${ }^{13} \mathrm{C}-\mathrm{NMR}$ spectra. $\left.{ }^{14}\right)$ The aqueous layer was neutralized by passage through Amberlite IRA-93 and elution with $\mathrm{H}_{2} \mathrm{O}$. This was followed by evaporation in vacuo to give a sugar fraction. The identity and configuration of the sugars were determined by comparison with authentic $\mathrm{D}-(+)$-fucose $\left(t_{\mathrm{R}}, 8.6 \mathrm{~min}\right)$ and $\mathrm{D}-(+)$-glucose $\left(t_{\mathrm{R}}, 11.2 \mathrm{~min}\right)$ on HPLC. HPLC conditions: column, Shodex RSpak DC-613 $(6.0 \times 150 \mathrm{~mm}$ i.d.); solvent, $\mathrm{CH}_{3} \mathrm{CN}-\mathrm{H}_{2} \mathrm{O}, 7: 3(\mathrm{v} / \mathrm{v})$; flow rate, $0.5 \mathrm{ml} / \mathrm{min}$; temperature, $70^{\circ} \mathrm{C}$; RI detection, Shodex RI-72 and chiral detection, JASCO OR-1590. The sugar fraction gave corresponding peaks of $\mathrm{D}-(+)$-fucose $\left(t_{\mathrm{R}}, 8.6 \mathrm{~min}\right)$ and D- $(+)$-glucose $\left(t_{\mathrm{R}}, 11.2 \mathrm{~min}\right)$.

Acid Hydrolysis of 3 A solution of $3(5.0 \mathrm{mg})$ in $5 \%$ aqueous $\mathrm{H}_{2} \mathrm{SO}_{4}$ $(1.0 \mathrm{ml})$ was heated at $110^{\circ} \mathrm{C}$ for $4 \mathrm{~h}$ under Ar. Water was added to the reaction mixture and the entire mixture was extracted with AcOEt. The organic layer was washed with water, dried over $\mathrm{MgSO}_{4}$ and evaporated. The residue was purified by silica gel open column chromatography $\left(\mathrm{MeOH} / \mathrm{CHCl}_{3}\right.$ gradient) to afford cincholic acid $(\mathbf{5}, 2.6 \mathrm{mg})$, as determined from ${ }^{1} \mathrm{H}$ - and ${ }^{13} \mathrm{C}$ NMR spectra. ${ }^{14)}$ The aqueous layer was neutralized by passage through Amberlite IRA-93 and elution with $\mathrm{H}_{2} \mathrm{O}$. This was followed by evaporation in vacuo to give a sugar fraction. The identity and configuration of the sugars, D-(+)-fucose and D-(+)-glucose, were determined by comparison with authentic sugars on HPLC under the same conditions as those described above.

Acid Hydrolysis of 4 A solution of $4(5.0 \mathrm{mg})$ in $5 \%$ aqueous $\mathrm{H}_{2} \mathrm{SO}_{4}$ $(1.0 \mathrm{ml})$ was heated at $110^{\circ} \mathrm{C}$ for $4 \mathrm{~h}$ under Ar. Water was added to the reaction mixture and the entire mixture was extracted with AcOEt. The organic layer was washed with water, dried over $\mathrm{MgSO}_{4}$ and evaporated. The residue was purified by silica gel open column chromatography $\left(\mathrm{MeOH} / \mathrm{CHCl}_{3}\right.$ gradient) to afford cincholic acid $(\mathbf{5}, 2.3 \mathrm{mg})$, as determined from ${ }^{1} \mathrm{H}$ - and ${ }^{13} \mathrm{C}$ NMR spectra. ${ }^{14)}$ The aqueous layer was neutralized by passage through Amberlite IRA-93 and elution with $\mathrm{H}_{2} \mathrm{O}$. This was followed by evaporation in vacuo to give a sugar fraction. The identity and configuration of the sugars, D-(+)-fucose and D-(+)-glucose, were determined by comparison with authentic sugars on HPLC. 
Acknowledgement One of the authors (M.K.) would like to thank Takeda Science Foundation for financial support.

\section{References}

1) Obregon L. E., "Cat's Claw, Uncaria Genus. Botanical, Chemical and Pharmacological Studies of Uncaria tomentosa (WILlD.) D.C. (Rubiaceae) and Uncaria guianensis (Aubl.) GMel.," Instituto de Fitoterapia Americano, Lima, 1995.

2) Cabieses F., "The Saga of the Cat's Claw," Via Lactea Editores, Lima, 1994.

3) Laus G., Keplinger K., Phyton, 43, 1-8 (2003) and references cited therein.

4) Aquino R., De Tommasi N., De Simone F., Pizza C., Phytochemistry, 45, 1035-1040 (1997) and references cited therein.

5) Wirth C., Wagner H., Phytomedicine, 4, 265-266 (1997) and references cited therein.

6) Sandoval M., Okuhara N. N., Zhang X.-J., Condezo L. A., Lao J., Angeles F. M., Musah R. A., Bobrowski P., Miller M. J. S., Phytomedicine, 9, 325-337 (2002) and references cited therein.

7) Sheng Y., Li L., Holmgren K., Pero R. W., Phytomedicine, 8, 275-
282 (2001) and references cited therein

8) Kang T.-H., Matsumoto K., Tohda M., Murakami Y., Takayama H., Kitajima M., Aimi N., Watanabe H., Eur. J. Pharmacol., 444, 39-45 (2002).

9) Kang T.-H., Murakami Y., Matsumoto K., Takayama H., Kitajima M., Aimi N., Watanabe H., Eur. J. Pharmacol., 455, 27-34 (2002).

10) Kitajima M., Hashimoto K., Yokoya M., Takayama H., Aimi N., Tetrahedron, 56, 547-552 (2000).

11) Kitajima M., Hashimoto K., Yokoya M., Takayama H., Sandoval M., Aimi N., J. Nat. Prod., 66, 320-323 (2003).

12) Kitajima M., Hashimoto K., Yokoya M., Takayama H., Aimi N., Chem. Pharm. Bull., 48, 1410-1412 (2000).

13) Kitajima M., Yokoya M., Takayama H., Aimi N., Nat. Med., 55, 308310 (2001).

14) Rumbero-Sanchez, A., Vazquez, P., Phytochemistry, 30, 623-626 (1991).

15) Lamidi M., Olliver E., Faure R., Debrauwer L., Nze-Ekekang L., Balansard G., Planta Med., 63, 284-285 (1997).

16) Aquino R., De Simone F., Pizza C., Cerri R., De Mello J. F., Phytochemistry, 27, 2927-2930 (1988).

17) Aquino R., De Simone F., Pizza C., Conti C., Steon M. L., J. Nat. Prod., 52, 679-685 (1989). 